\title{
IL-4-polarized BV2 microglia cells promote angiogenesis by secreting exosomes
}

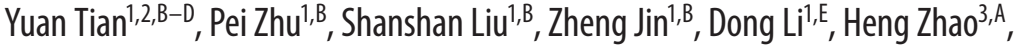 \\ Xun Zhu ${ }^{1, A}$, Chang Shu ${ }^{4, C}$, Dongmei Yan ${ }^{1, A, D-F}$, Zehua Dong ${ }^{5, A, F}$ \\ ${ }^{1}$ Department of Immunology, College of Basic Medical Sciences, Jilin University, Changchun, China \\ ${ }^{2}$ Key Laboratory of Molecular Enzymology and Engineering under the Ministry of Education, College of Life Sciences, Jilin University, Changchun, China \\ ${ }^{3}$ Department of Neurosurgery, Stanford University, USA \\ ${ }^{4}$ Department of Obstetrics, First Hospital of Jilin University, Changchun, China \\ ${ }^{5}$ Intensive Care Unit, the Affiliated Hospital of Qingdao University, China \\ A - research concept and design; $\mathrm{B}$ - collection and/or assembly of data; $\mathrm{C}$ - data analysis and interpretation; \\ $D$ - writing the article; $E$ - critical revision of the article; $F$ - final approval of the article
}

Address for correspondence

Dongmei Yan

E-mail:dmyan@jlu.edu.cn

\section{Funding sources}

The study was supported by funding from the International Science and Technology Cooperation Base of the Jilin Province Science and Technology

Department (No. 20150414035GH) and the National Natural Science Foundation of China ( $N$ o. 81501279).

Conflict of interest

None declared

Received on January 19, 2018

Reviewed on January 30, 2018

Accepted on May 29, 2018

Published online on January 22, 2019

Cite as

Tian Y, Zhu P, Liu S, et al. IL-4-polarized BV2 microglia cells promote angiogenesis by secreting exosomes. Adv Clin Exp Med. 2019;28(4):421-429. doi:10.17219/acem/91826

DOI

$10.17219 /$ acem/91826

\section{Copyright}

Copyright by Author(s)

This is an article distributed under the terms of the

Creative Commons Attribution Non-Commercial License

(http://creativecommons.org/licenses/by-nc-nd/4.0/)

\begin{abstract}
Background. The microglia cell transfer has been shown to play a protective role in ischemic stroke. Microglia cells may play this nerve-protective role via the promotion of angiogenesis. However, the underlying mechanisms are largely unknown and need further investigation.

Objectives. The aim of this study was to investigate the pro-angiogenesis effects of unpolarized, interleukin 4 (IL-4)-polarized or lipopolysaccharide (LPS)-polarized BV2 microglia cells both in vivo and in vitro. We also investigated the potential mechanisms of these pro-angiogenesis effects.

Material and methods. BV2 cells were polarized using phosphate-buffered saline (PBS), LPS or IL-4, respectively. The gene expression pattern was analyzed with reverse transcription polymerase chain reaction (RT-PCR). The transfer of polarized BV2 cells was performed with an intravenous injection into mice 45 min after the middle cerebral artery (MCA) occlusion. Angiogenin expression was assessed with immunofluorescence. We also examined the angiogenesis effect of polarized BV2 cells and their exosomes through 3-dimensional co-cultures in vitro. Finally, the microRNA (miRNA) profiles of exosomes released by BV2 cells under different polarization conditions were examined using miRNA microarray.
\end{abstract}

Results. The IL-4-polarized BV2 transplantation promoted angiogenin expression in the ischemic brain. Interleukin 4-polarized microglia increased the tube formation of endothelial cells by secreting exosomes. The miRNA profiles of exosomes released by BV2 cells under different polarization conditions were different. Exosomes from IL-4-polarized BV2 cells contained higher amounts of miRNA-26a compared to those from the LPS-polarized and unpolarized BV2 cells.

Conclusions. Interleukin 4-polarized microglia cells might ameliorate the damage caused by ischemic stroke by promoting angiogenesis through the secretion of exosomes containing miRNA-26a.

Key words: angiogenesis, exosomes, microglia, interleukin 4 


\section{Introduction}

Stroke is the second leading cause of death worldwide. It is most often caused by a thrombus or an embolus in the middle (MCA) or anterior cerebral artery (ACA), where an infarct develops after a few minutes of ischemia. Microglia are a type of neuroglia (glial cells) located throughout the central nervous system; they account for $10-15 \%$ of all cells within the brain and spinal cord. Microglia are also a type of resident macrophage cells, and are the first responding cells during the development of a stroke. ${ }^{1,2}$ There have been reports indicating that transplanting unstimulated HMO6 human microglial cells could protect animals from neural damage in a stroke by reducing neuronal cell death. ${ }^{3}$

Microglia/macrophages are known to have distinct phenotypes with various and even opposing functions. It has recently been reported that microglia with the M2-like phenotype (interleukin 4 (IL-4)-polarized microglia) are initially recruited at the injury site after the middle cerebral artery occlusion (MCAO), but they polarize to the M1like phenotype (lipopolysaccharide (LPS)-polarized microglia) at a later stage. ${ }^{4,5}$ The M2-like microglia which are induced by type II cytokines like IL-4 and IL-13 could secret cytokines that promote regenerative processes and neurogenesis while suppressing type I immunity; thus, the M2-like microglia could be beneficial in spinal cord injury and in stroke. ${ }^{6}$ Moreover, IL-4 has been proven to be essential for the treatment of ischemic brain damage, as well as for polarizing microglia/macrophages to a M2-like subset. ${ }^{7}$ Therefore, manipulating the polarization of microglia/macrophages might be a better treatment method not only for experimental stroke but in clinical treatment as well. ${ }^{8,9}$ A direct injection of M2-type bone marrowderived macrophages (BMDM) after ischemia did not lead to a significant improvement, ${ }^{10}$ but this may be due to different causes of stroke, and different micro-environments may drive BMDM to different phenotypes with opposing functions under hypoxia conditions. ${ }^{11}$ In short, the therapeutic effect of a direct M2 microglia/macrophages transplantation has not yet been proven. Polarizing resident microglia/macrophages in vivo may be a more suitable approach to the treatment of stroke.

Exosomes are small vesicles originating from the fusion of multivesicular bodies with a plasma membrane. The contents of exosomes, including proteins, mRNAs and microRNAs (miRNAs), play a crucial role in the activation, polarization or inhibition of immune cells. Recent findings indicate that exosomes derived from mesenchymal stromal cells significantly improved functional recovery by promoting angiogenesis in stroke rats. ${ }^{12}$ Angiogenesis stimulates other downstream events, including neurogenesis, synaptogenesis, and neuronal and synaptic plasticity, which are all involved in the long-term repair and restoration process of the brain after an ischemic event. Particularly, recent studies have shown that the conditioned medium from metformin-treated BV2 cells can promote angiogenesis in vitro. ${ }^{13}$ Whether IL-4-polarized microglia may promote angiogenesis by secreting exosomes needs further investigation.

In this work, we transferred polarized microglia to the brain in a mouse stroke model to determine the protective effect of microglia in vivo; we also investigated the underlying mechanisms in vitro by co-culturing endothelial cells with polarized microglia cells or exosomes secreted by those cells. Our results showed that M2-like microglia could protect the central nervous system, probably through the secretion of pro-angiogenesis exosomes.

\section{Material and methods}

\section{BV2 cell culture and polarization}

Mouse microglial cell line BV2 cells were cultured in a complete medium (Roswell Park Memorial Institute (RPMI) 1640 with L-glutamine, supplemented with $10 \%$ fetal bovine serum (FBS), $100 \mathrm{U} / \mathrm{mL}$ penicillin and $100 \mu \mathrm{g} / \mathrm{mL}$ streptomycin) (North China Pharmaceutical Group Corp., Shijiazhuang, China). In order to eliminate exosomes, FBS was ultracentrifuged at 120,000 g for 90 min using a Ti70 rotor (Optima ${ }^{\mathrm{TM}} \mathrm{LE}-80 \mathrm{~K}$ Ultracentrifuge; Beckman Coulter Life Sciences, Indianapolis, USA) before being added to the medium. The cells were maintained at $37^{\circ} \mathrm{C}$ in a $5 \%$ $\mathrm{CO}_{2}$ incubator. BV2 cells were seeded at $1 \times 10^{6}$ per well in a $25 \mathrm{~cm}^{2}$ plate for $24 \mathrm{~h}$ before being stimulated with LPS $(1 \mu \mathrm{g} / \mathrm{mL})$ or murine IL- $4(25 \mathrm{ng} / \mathrm{mL})$ for $48 \mathrm{~h}$ to generate classically activated macrophages (M1) or alternatively activated macrophages (M2), respectively.

\section{Gene expression of polarized BV2 cells by reverse transcription polymerase chain reaction}

For the reverse transcription polymerase chain reaction (RT-PCR) assay, BV2 cells were seeded in a $25 \mathrm{~cm}^{2}$ flask and polarized with LPS $(1 \mu \mathrm{g} / \mathrm{mL})$ and IL- $4(25 \mathrm{ng} / \mathrm{mL})$ to generate M1 and M2, respectively. After polarization, total RNA was extracted with TRIzol ${ }^{\mathrm{TM}}$ Reagent (Invitrogen, Carlsbad, USA), following the manufacturer's protocol, and then reverse-transcribed into complementary DNA (cDNA) using the SuperScript ${ }^{\circledR}$ III First-Strand Synthesis System for RT-PCR (Invitrogen). The gene primers are shown in Table 1. The parameters for RT-PCR were as follows: denaturing at $94^{\circ} \mathrm{C}$ for $3 \mathrm{~min}$, followed by 25 cycles of $94^{\circ} \mathrm{C}$ for $30 \mathrm{~s}, 51^{\circ} \mathrm{C}$ for $1 \mathrm{~min}$ and $72^{\circ} \mathrm{C}$ for $1 \mathrm{~min}$, and then extension at $72^{\circ} \mathrm{C}$ for $2 \mathrm{~min}$.

\section{Middle cerebral artery occlusion model and transplantation of microglia}

The animal procedures were approved by the Stanford Institutional Animal Care and Use Committee (Stanford University, USA) and were in accordance with the National 
Table 1. Forward and reverse gene primers sequences

\begin{tabular}{|l|c|}
\hline Gene & Primers \\
\hline iNOS (forward) & ATGGCAACATCAGGTCGG \\
iNOS (reverse) & GCACAACTGGGTAACTCC \\
\hline TNF- (forward) & ACTGAACTTCGGGGTATCG \\
TNF- (reverse) & CCACTTGGTGGTTTGTACG \\
\hline Argl (forward) & CAGTCTGGCAGTTGGAAGC \\
Argl (reverse) & GGTTGTCAGGGGAGTGTTG \\
\hline TGF- $\beta$ (forward) & GGACTACTACGCCAAAGAAG \\
\hline TGF- $\beta$ (reverse) & TCAAAAGACAGCCACTCAGG \\
\hline GAPDH (forward) & GACTTCAACAGCAACTCCCACTC \\
GAPDH (reverse) & TAGCCGTATTCATTGTCATACCAG \\
\hline VEGF (forward) & CTGCTGTAACGATGAAGCCCTG \\
VEGF (reverse) & GCTGTAGGAAGCTCATCTCTCC \\
\hline HGF (forward) & CTCCTGAAGGCTCAGACTTGGT \\
\hline HGF (reverse) & CCAGAAGTAAATATGCAAGTGG \\
\hline FGF (forward) & AAGCGGCYCYACYGCAAGAACG \\
FGF (reverse) & CCTTGATAGACACAACTCCTCTC \\
\hline EGF (forward) & ACTGGTGTGACACCAAGAGGTC \\
EGF (reverse) & CCACAGGTGATCCTCAAACACG \\
\hline PGF (forward) & TGCTGTGGTGATGAAGGTCTGC \\
\hline PGF (reverse) & GCATTCACAGAGCACATCCTGAG \\
\hline PDGF (forward) & GTGGTCCTTACCGTCATCTCTC \\
PDGF (reverse) & GTGGAGTCGTAAGGCAACTGCA \\
\hline MMP2 (forward) & CAAGGATGGACTCCTGGCACAT \\
MMP2 (reverse) & TACTCGCCATCAAGCGTTCCCAT \\
\hline MMP9 (forward) & GCTGACTACGATAAGGACGGCA \\
MMP9 (reverse) & TAGTGGTGCAGGCAGAGTAGGA \\
\hline
\end{tabular}

Institutes of Health (NIH) Guidelines for the Care and Use of Laboratory Animals. All efforts were made to minimize the number of animals used and their suffering.

Male C57BL/J (20 $\pm 2 \mathrm{~g})$ mice were purchased from The Jackson Laboratory (Bar Harbor, USA). Transient MCAO was induced using the method of intraluminal vascular occlusion described in our previous studies. ${ }^{14}$ Briefly, the mice were initially anesthetized with $5 \%$ isoflurane, and maintained at $1-2 \%$ during the surgery in a $70 \% \mathrm{~N}_{2} \mathrm{O}$ and $30 \% \mathrm{O}_{2}$ mixture using a face mask. In order to maintain the body core temperature of the mice at $37 \pm 0.5^{\circ} \mathrm{C}$, a surface heating pad was used and the temperature was monitored by a rectal probe during the entire procedure. Then, the left common carotid artery, external carotid artery and internal carotid artery were surgically exposed by a ventral midline neck incision. The mice were subjected to MCAO with a 6-0 nylon monofilament suture (Doccol Corp., Sharon, USA), coated with silicone. At 45 min after MCAO, the occluded animals were re-anesthetized, the nylon monofilament suture was removed and the end of the external carotid artery was tied. The mice were allowed to wake up from anesthesia and returned to the cages.

The mice were randomly divided into 4 groups as follows ( $\mathrm{n}=5-7$ mice per group): 1 . vehicle treatment; 2 . treatment with unpolarized microglia (the control group); 3. treatment with LPS-polarized microglia; and 4. treatment with IL-4-polarized microglia. One million microglia cells in $0.1 \mathrm{~mL}$ of phosphate-buffered saline (PBS) were administered to each mouse with vein infusion immediately after the transient MCAO; the vehicle-treated mice were injected with an equal volume of PBS.

\section{Immunofluorescence staining}

At 2 days after MCAO, 3 groups of 5 experimental animals each were sacrificed and their organs were fixed by transcardial perfusion with PBS. The brain tissues were immersed in $4 \%$ paraformaldehyde (PFA) in $0.1 \mathrm{~mol} / \mathrm{L} \mathrm{PBS}$ ( $\mathrm{pH}$ 7.4) for $48 \mathrm{~h}$. The brain tissues were cut into equal samples (thickness: $50 \mu \mathrm{m}$ ).

The slides were fixed with methyl alcohol and acetone (1:1) for $10 \mathrm{~min}$, and then washed 3 times with PBS. After washing, the slides were blocked with $5 \%$ bovine serum albumin (BSA) and incubated overnight with antiangiogenin Ab (diluted 1:200; Abcam, Cambridge, USA). The slides were washed 3 times in a wash buffer (PBS with $0.05 \%$ Tween ${ }^{\circledR} 20$ (Institute of Chemical Technology, Beijing, China) for 15 min each time. After washing, the secondary antibody coupled with fluorescence was added and incubated for $2 \mathrm{~h}$ at room temperature. Then, 4',6-diamidino-2-phenylindole (DAPI) was added for staining for $5 \mathrm{~min}$, followed by washing. The slides were sealed with Fluoromount ${ }^{\mathrm{TM}}$ (Sigma-Aldrich, St. Louis, USA) and observed with confocal laser scanning microscopy using an Axio Vert inverted scanning microscope (Carl Zeiss AG, Oberkochen, Germany).

\section{Endothelial cell co-culture with polarized BV2 cells and tube formation assay}

In order to analyze the mechanism of the therapeutic effect of IL-4-polarized microglia and to investigate whether these cells can promote angiogenesis, we introduced a co-culture of endothelial cells and microglia. For all the in vitro experiments, the human umbilical vein endothelial cells (HUVEC) were used. The cells were cultured in Dulbecco's Modified Eagle Medium (DMEM), supplemented with $10 \%$ fetal calf serum (FCS), $100 \mathrm{U} / \mathrm{mL}$ penicillin and $100 \mu \mathrm{g} / \mathrm{mL}$ streptomycin. Then, 48-well plates were filled with $150 \mu \mathrm{L}$ Matrigel ${ }^{\circledR}$ (BD Biosciences, Franklin Lakes, USA) and allowed to solidify at $37^{\circ} \mathrm{C}$ for 30 min. Subsequently, BV2 microglia subsets $\left(4 \times 10^{3}\right.$ cells/well) were co-incubated with the HUVEC $\left(4 \times 10^{4}\right.$ cells/well). After $24 \mathrm{~h}$, network structures were analyzed at $\times 40$ magnification using the AxioVision Microscopy software (Carl Zeiss AG) and photographed with a digital camera (ECLIPSE TS 100-F; Nikon, Tokyo, Japan). ${ }^{15,16}$ The number of tubes per picture was counted using the Image J program (National Intitutes of Health, Bethesda, USA). 


\section{Assessment of vascular endothelial growth factor secreted by polarized BV2 cells}

The supernatants of the polarized BV2 cells were collected after $48 \mathrm{~h}$ of LPS and IL- 4 stimulation. Then, the concentration of vascular endothelial growth factor (VEGF) secreted by the polarized BV2 cells was detected using the SMMV00 enzyme-linked immunosorbent assay (ELISA) kit (R\&D Systems, Inc., Minneapolis, USA). The detection of the concentration of VEGF in the control, M1 and M2 supernatants was repeated 3 times.

\section{Isolation and identification of microglia exosomes}

The microglia exosome isolation procedures were performed at $4^{\circ} \mathrm{C}$ as described in the literature, using an exosome extraction kit (System Biosciences, Palo Alto, USA). Briefly, the cell supernatants were centrifuged at $3000 \mathrm{~g}$ for $15 \mathrm{~min}$ to remove cells and cell debris; the supernatants were added to an exosome extraction reagent and mixed gently. After $12 \mathrm{~h}$ at $4{ }^{\circ} \mathrm{C}$, the mixture was centrifuged at $10,000 \mathrm{~g}$ for $30 \mathrm{~min}$; the pellets were collected and resuspended in $50-100 \mu \mathrm{L}$ of PBS, and used for the analysis of the exosome-enriched fraction. For the transmission electron microscopy (TEM) morphology investigation, the pellets obtained by this process were subjected to uranyl acetate negative staining on the formvar/carbon-coated 400-mesh copper electron microscopy grids (FCF400-Cu; Electron Microscopy Sciences, Hatfield, USA). Twenty microliters of the sample were applied to the grid and incubated for $1 \mathrm{~min}$ at room temperature, and then the excess solution on the grid was wicked off and dried for 30 min with filter papers. An equal part of $10 \%$ uranyl acetate was added to the grid for $1 \mathrm{~min}$ for negative staining. The preparations obtained were examined at $70 \mathrm{kV}$ with a Philips 208 electron microscope (Philips Healthcare, Bothell, USA) with the DigitalMicrograph ${ }^{\mathrm{TM}}$ (Gatan, Inc., Pleasanton, USA). Western blot was used to identify TSG101, CD81 and CD63 (primary antibody, 1:200; Santa Cruz Biotechnology, Inc., Santa Cruz, USA), the specific exosomal protein markers. The protein concentrations of the exosome preparations were determined using the micro bicinchoninic acid protein assay (Thermo Fisher Scientific, Lafayette, USA).

\section{Tube formation by microglia exosomes}

Exosomes from other cells, such as multiple myeloma cells and mesenchymal stem cells, can promote angiogenesis. The effect of exosomes from IL-4-polarized microglia on angiogenesis has not been clear. The HUVEC were cultured as described above, then 48-well plates were filled with $150 \mu \mathrm{L}$ Matrigel (BD Biosciences) and allowed to solidify at $37^{\circ} \mathrm{C}$ for $30 \mathrm{~min}$. The exosomes were separated by the method described above. Briefly, the exosomes from $5 \times 10^{6}$ polarized cells were co-incubated with the HUVEC. After $12 \mathrm{~h}$, network structures were analyzed at $\times 40$ magnification using the AxioVision Microscopy software (Carl Zeiss AG) and photographed with a digital camera (Nikon). The number of tubes per image was counted using Image (NIH).

\section{RNA extraction and microRNA array}

RNA was extracted with TRIzol (Invitrogen) according to the manufacturer's protocol, and the Nanodrop ${ }^{\mathrm{TM}}$ Spectrophotometer (Thermo Fisher Scientific, Waltham, USA) was used to assess the RNA present. The samples extracted from M0, M1 and M2 type microglia exosomes were then delivered to GMINIX Co. (Shanghai, China). Commercial mouse miRNA microarrays, containing 1,908 mouse mature microRNAs from the Sanger mirBase database v. 20.0 (2 probes for each miRNA on each chip) were used to analyze the expression of miRNA in diferent types of BV2 cells. The tagged miRNAs were purified and hybridized with the GMINIX microRNA Microarray-Single according to the manufacturer's instructions. After the hybridization, the chips were subjected to a stringent wash and fluorescence data were collected using the GeneChip Scanner 3000 (Thermo Fisher Scientific, Waltham, USA); the chips were scanned at a pixel size of $10 \mu \mathrm{M}$ with Cyanine 3 (Cy3) Gain at $460 \mathrm{~nm}$ and Cyanine 5 (Cy5) Gain at $470 \mathrm{~nm}$ scanning. Equal RNA from 6 individual cell samples with the same treatment was mixed and each mixture sample was repeated twice.

The data was shown as mean \pm standard deviation (SD). The p-values were calculated using Student's t-test and post hoc test for multiple comparisons.

\section{Results}

\section{Interleukin 4-polarized BV2 cells upregulated the expression of angiogenin in the ischemic brain}

Angiogenin is a potent angiogenic growth factor that degrades the basement membrane, thereby facilitating cell invasion and migration. To check whether BV2 cells could induce angiogenesis by producing angiogenin, we polarized BV2 cells with either LPS or IL-4. The gene expression pattern was analyzed by RT-PCR (Fig. 1). We also detected the expression of angiogenin in the ischemic brain after IL-4-polarized BV2 treatment using immunofluorescence staining. The results showed that IL-4-polarized microglia significantly increased the expression of angiogenin and vascular density after 14 days compared with the control mice (Fig. 2). 
A

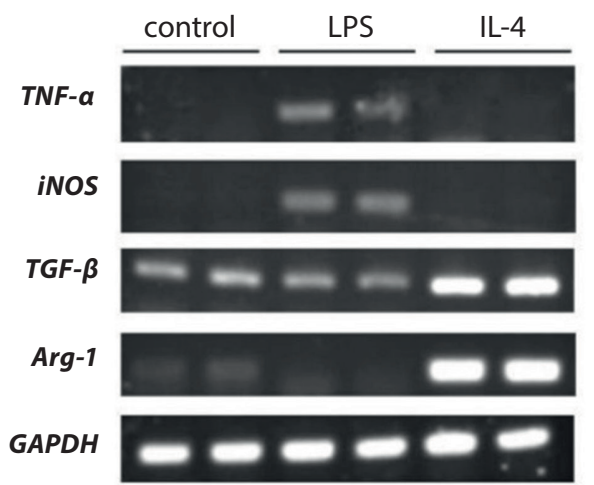

B

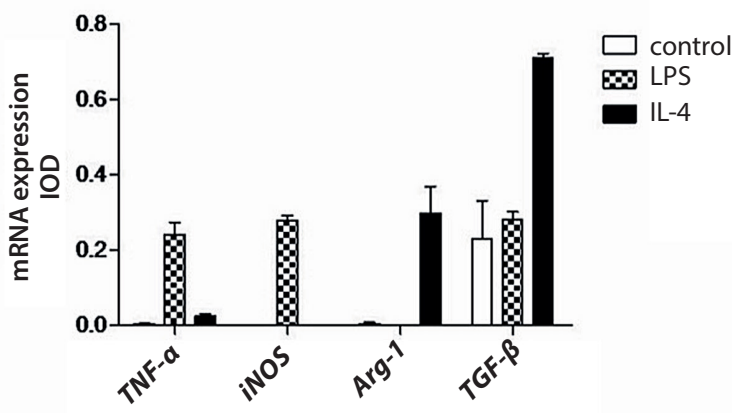

Fig. 1. Gene expression of polarized BV2 cells

A. Reverse transcription polymerase chain reaction (RT-PCR) results showing that BV2 express M1 markers (TNF- $a$ and iNOS) or M2 markers (Arg-1 and TGF- $\beta$ )

B. Quantitative data for the expression of TNF- $\alpha$, iNOS, Arg-1, and TGF- $\beta$

Data is presented as mean \pm standard deviation (SD) from triplicates; Arg-1 - arginase 1; GAPDH - glyceraldehyde 3-phosphate dehydrogenase; IL-4 - interleukin 4; INOS - inducible nitric oxide synthase; IOD - integrated optical density; LPS - lipopolysaccharide; TGF- $\beta$ - transforming growth factor $\beta$; TNF- $a$ - tumor necrosis factor $\alpha ;$ M1 - classically activated macrophages; M2 - alternatively activated macrophages

A

2 days

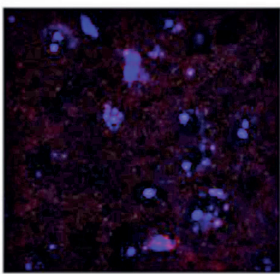

M2

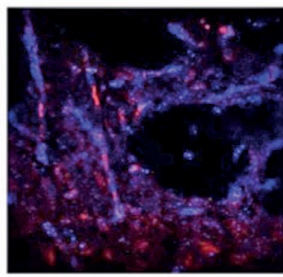

A

HUVEC

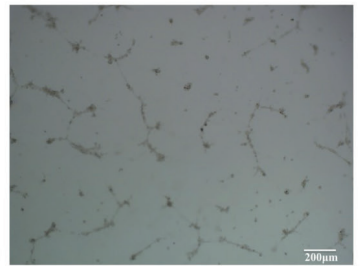

HUVEC + M1

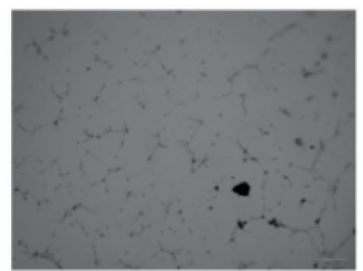

14 days
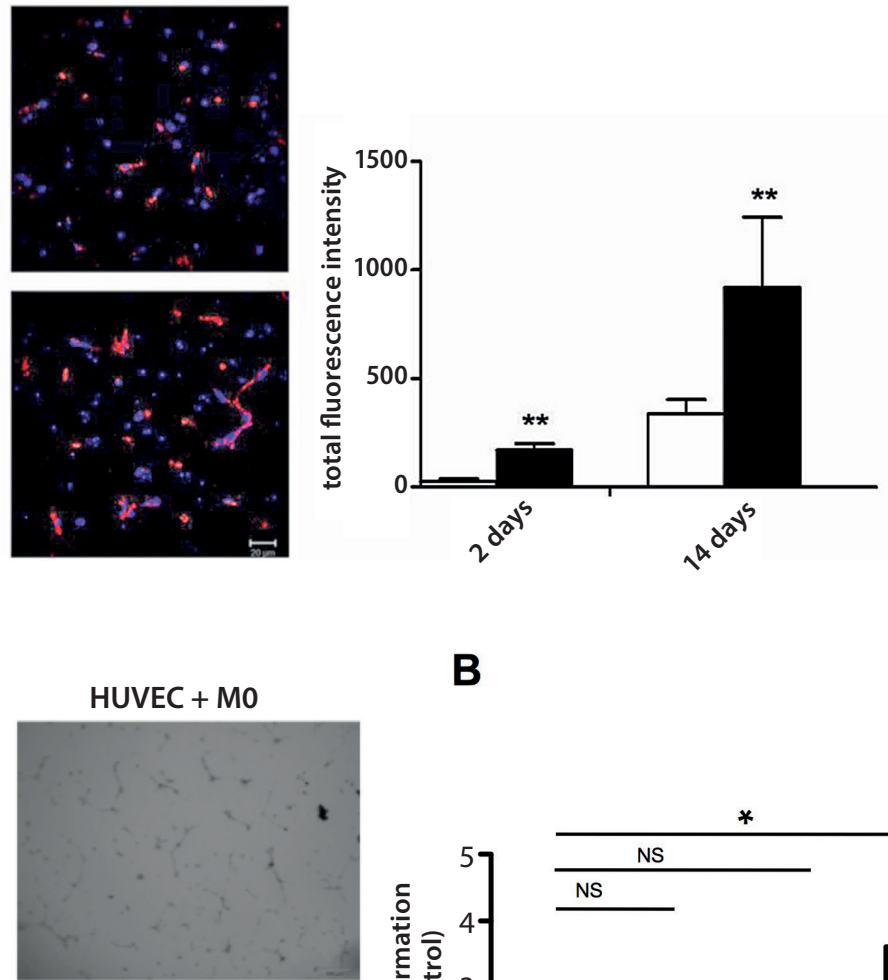

HUVEC + M2

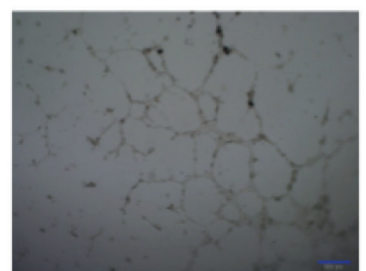

B

B

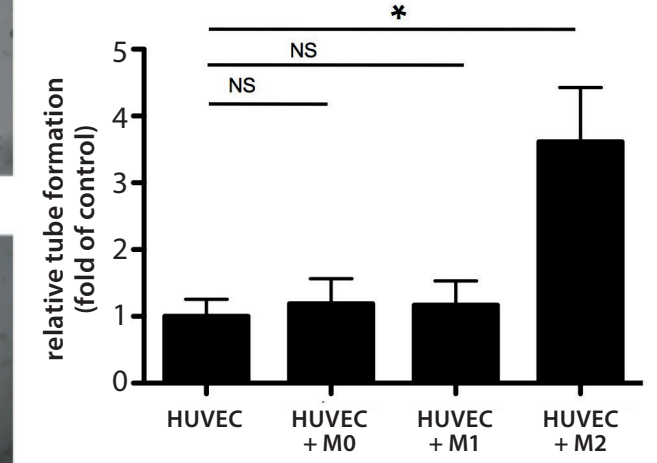

Fig. 2. Expression of angiogenin in ischemic mice increased 14 days after the M2 microglia transplantation

A. Immunostaining of angiogenin in the ischemic brain on day 2 and day 14 after control and interleukin 4

$\square$ M0 (IL-4)-polarized BV2 treatment (4, 6-diamidino2-phenylindole (DAPI): blue; angiogenin: red; $n=3$ per group; the scale bar represents $20 \mu \mathrm{m}$ ) B. Total fluorescence of angiogenin in the ischemic brain on day 2 and day 14

M0 - control; M2 - IL-4 -polarized BV2 treatment: ** $\mathrm{p}<0.01$ compared to the M0 group.

Fig. 3. Interleukin 4-polarized microglia promoted angiogenesis in vitro. BV2 microglia subsets $\left(4 \times 10^{3}\right.$ cells/well) were co-incubated with human umbilical vein endothelial cells (HUVEC) $\left(4 \times 10^{4}\right.$ cells/well) for $24 \mathrm{~h}$

A. Network structures were analyzed at $\times 40$ magnification and photographed with a digital camera $(n=3$ per group; the scale bar represents $200 \mu \mathrm{m}$ )

B. The number of tubes per picture was counted using ImageJ software

M1 - LPS-polarized BV2 treatment: * $p<0.05$ compared to the HUVEC group; NS - statistically nonsignificant. 
A

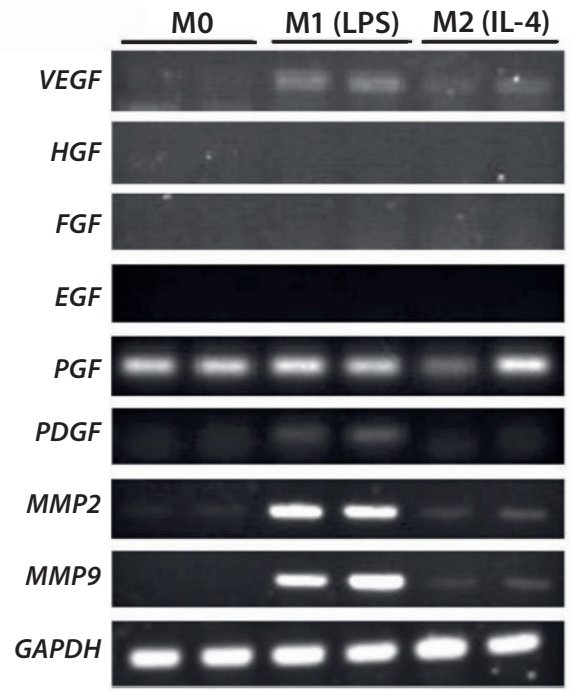

B

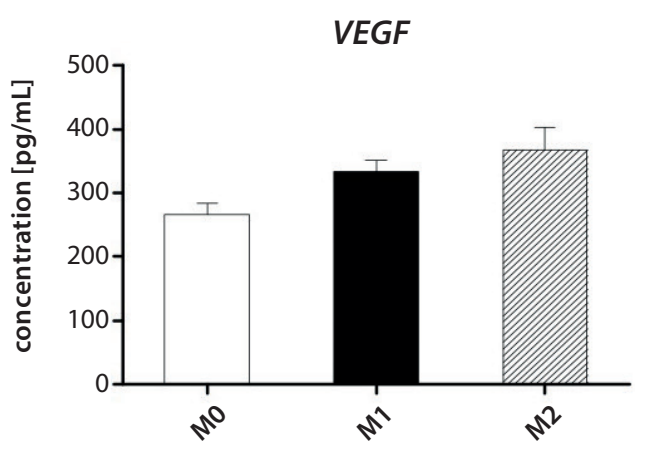

Fig. 4. Angiogenesis-related gene expression of polarized BV2 cells. The expression of angiogenesis-related genes was detected with RT-PCR and the release of VEGF was detected in the culture supernatants of polarized BV2 cells using enzyme-linked immunosorbent assay (ELISA)

A. Expression of genes related to angiogenesis in M0, M1 and M2 BV2 cells

B. Concentrations of VEGF in the culture supernatants of M0, M1 and M2 BV2 cells; there were no significant differences between these cells ( $p$ > 0.05)

EGF - epidermal growth factor; FGF - fibroblast growth factor; HGF - hepatocyte growth factor; MMP2 - matrix metalloproteinase 2; MMP9 - matrix metalloproteinase 9; PDGF - platelet-derived growth factor; PGF - placental growth factor; VEGF - vascular endothelial growth factor.

A

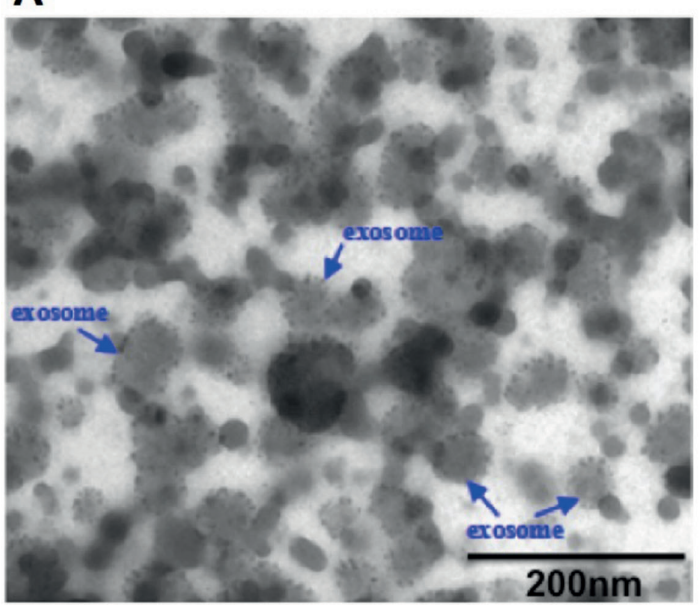

B

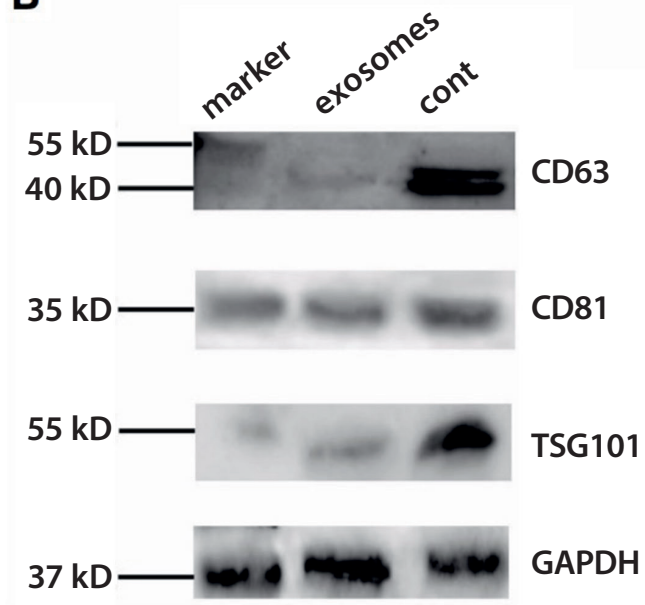

Fig. 5. Characteristics of exosomes

A. Transmission electron micrographs of the exosomes derived from BV2 cells stimulated by IL-4 (the scale bar represents $200 \mathrm{~nm}$ ) B. Western blotting analysis for exosome markers CD63, CD81 and TSG101

cont - positive control.

\section{Interleukin 4-polarized BV2 cells increased the tube formation in vitro}

To study the role of macrophage subsets in angiogenesis, we performed in vitro tube formation assays using a coculture of endothelial cells and macrophages on a Matrigel base. Culturing endothelial cells in these settings led to the formation of tubular structures after $24 \mathrm{~h}$. Adding IL-4-polarized BV2 cells to endothelial cells increased the number of tubes as compared to the control situation (Fig. 3).

\section{Interleukin 4-polarized BV2 cells promote the tube formation by secreting exosomes}

In order to ascertain the mechanism by which IL-4-polarized BV2 cells promote angiogenesis, we first detected the gene expression of secreted growth factors related to angiogenesis, such as $V E G F$, hepatocyte growth factor $(H G F)$, fibroblast growth factor $(F G F)$, epidermal growth factor $(E G F)$, placental growth factor $(P G F)$, platelet-derived growth factor (PDGF), matrix metalloproteinase 2 (MMP2), and matrix metalloproteinase 9 (MMP9). Although the VEGF gene expression increased in the IL-4-polarized BV2 group compared with the control group, its level was lower than in the LPS-polarized group (Fig. 4A). There were no significant differences in the ELISA results for VEGF between the control culture, LPS-polarized BV2 cells and IL-4-polarized BV2 cells (Fig. 4B). The expression of other genes showed no significant increase in the IL-4-polarized BV2 group compared with the LPS-polarized group (Fig. 4A). These results showed that the tube formation of endothelial cells 
A

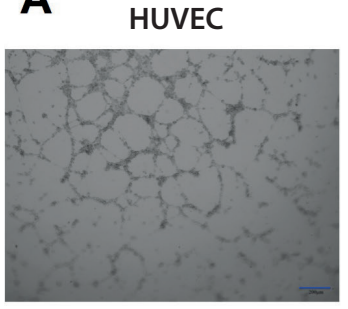

HUVEC + M1 exo

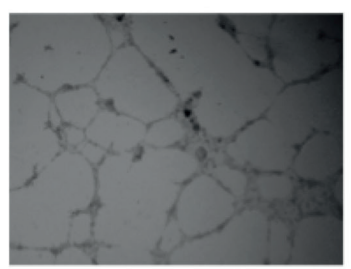

HUVEC + MO exo

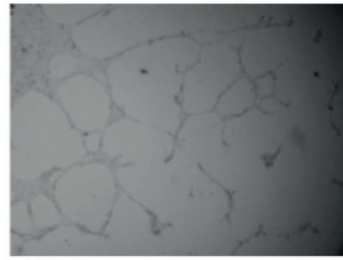

HUVEC + M2 exo

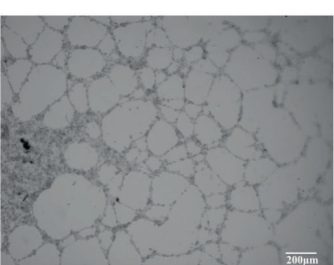

B

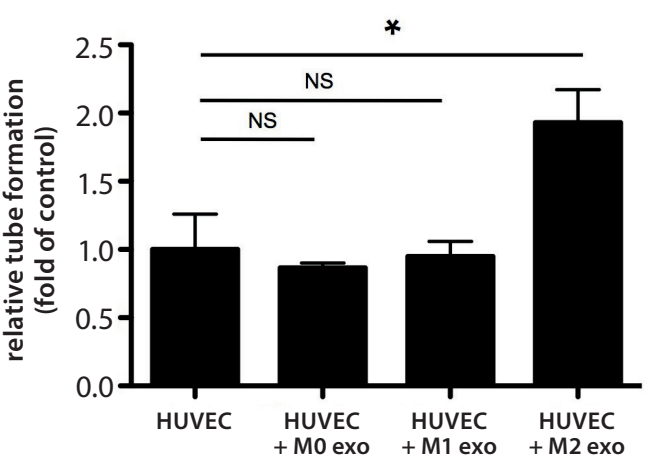

Fig. 6. IL-4-polarized microglia exosomes promoted angiogenesis in vitro. The exosomes from $5 \times 10^{6}$ polarized cells were co-incubated with HUVEC cells for $12 \mathrm{~h}$

A. Network structures were analyzed at $\times 40$ magnification and photographed with a digital camera ( $n=3$ per group; the scale bar represents $200 \mu m$ ) B. The number of tubes per picture was counted using ImageJ software

exo - exosomes; * $p<0.05$ compared to the HUVEC group; NS - statistically nonsignificant.

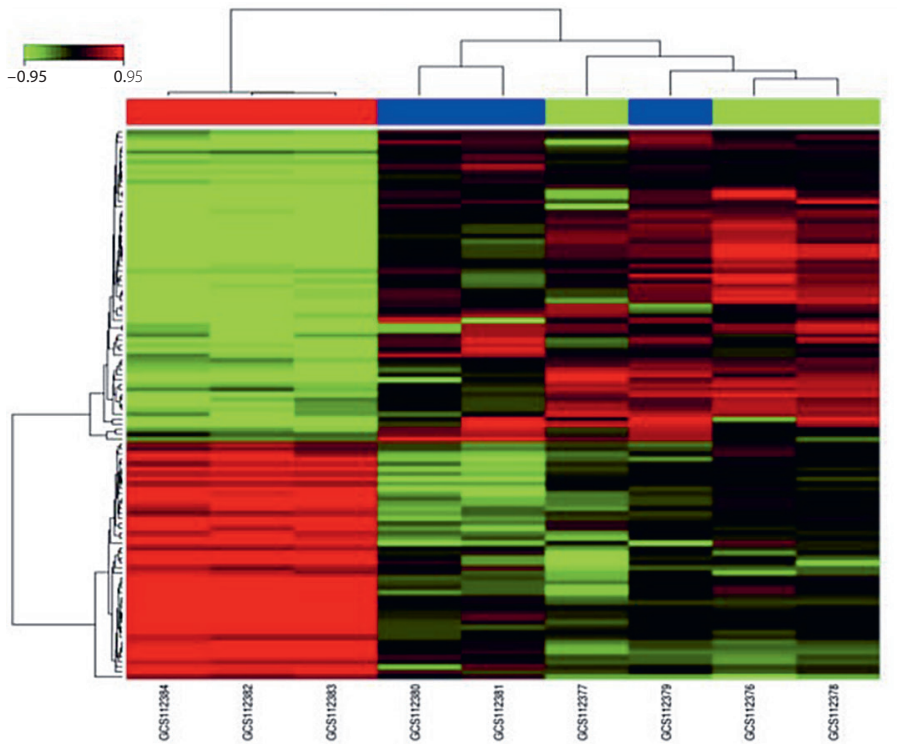

promoted by IL-4-polarized macrophages was not related to the secretion of VEGF, HGF, EGF, PGF, PDGF, MMP2, and MMP9.

We next investigated the exosomes secreted by polarized BV2 cells in terms of size, ultrastructures and quantity, and then analyzed their activities promoting angiogenesis. Transmission electron microscopy revealed that the size of the exosomes was about $50-100 \mathrm{~nm}$ and each vesicle showed the classic cup-shaped appearance (Fig. 5A). Western blotting results showed the expression of common exosome markers like CD63, CD81 and TSG101 (Fig. 5B). Then, a tube formation assay was performed with exosomes from different microglia subsets. In comparison with the controls, the exosomes from IL-4-polarized BV2 cells stimulated the tube formation: the quantities of tubes
Fig. 7. Heat map of microRNA expression array of M0, M1 and M2 microglia-derived exosomes. Mo-exo An intensity key is given next to the heat map. M1-exo Each sample is represented as an average M2-exo of duplicates increased significantly (Fig. 6). There was no difference between the LPS-polarized group and the controls. This showed that the exosomes from IL-4-polarized BV2 microglia had pro-angiogenic properties.

The promotion of angiogenesis by the M2-type BV2 cells may be due to the secretion of miRNA-26a. Exosomes have been shown to play an important role in the regulation of cell activities. Recent studies have also shown that the content of exosomes, like miRNA, could enter a recipient cell once the exosome membrane fuses with the cell membrane. In this work, we analyzed the different miRNA profiles of unpolarized, LPS-polarized and IL-4-polarized BV2 cells (Fig. 7). We found that miRNA-26a, which has been shown to have angiogenic properties, was selectively upregulated by IL-4 polarization (Table 2$).{ }^{17}$ 
Table 2. MicroRNAs of exosomes released by M0-, M1- and M2-type microglia

\begin{tabular}{|l|c|c|c|}
\hline Transcript ID & Mean signal of group M0 & Mean signal of group M1 & Mean signal of group M2 \\
\hline miR-466b-3p & 5.521937 & 4.806909 & 2.125578 \\
\hline miR-466c-3p & 5.521937 & 4.806909 & 2.125578 \\
\hline miR-466p-3p & 5.521937 & 4.806909 & 7.226154 \\
\hline miR-8117 & 9.017336 & 8.827255 & 6.680118 \\
\hline miR-151-5p & 8.958132 & 8.5831889 & 13.094678 \\
\hline miR-3620-5p & 11.610691 & 11.805563 & 13.408188 \\
\hline miR-7221-3p & 12.137256 & 12.323571 & 11.439959 \\
\hline miR-677-3p & 9.540374 & 9.610824 & 7.637256 \\
\hline miR-1956 & 7.252635 & 7.637256 & 2.048182 \\
\hline miR-6360 & 4.987463 & 4.509745 & 12.896675 \\
\hline miR-6970-5p & 11.378213 & 11.735456 & 13.65795 \\
\hline miR-346-3p & 12.529241 & 12.37427 & 7.642079 \\
\hline miR-26a-5p & 9.300976 & 8.937378 & 12.375583 \\
\hline miR-7033-5p & 10.858716 & 10.955668 & 8.055301 \\
\hline miR-702-5p & 9.151745 & 9.149582 & 7.689586 \\
\hline miR-15b-5p & 9.812329 & 9.292808 & 12.264028 \\
\hline miR-7235-5p & 11.251865 & 11.324551 & 5.388706 \\
\hline miR-1940 & 7.170623 & 7.549589 & 6.029101 \\
\hline miR-7049-5p & 7.799654 & 7.473127 & 2.280584 \\
\hline miR-466a-5p & 5.572361 & 5.641108 & \\
\hline
\end{tabular}

\section{Discussion}

Our study offers a few points on the therapeutic effect of IL-4-polarized microglia in the acute and chronic phases of ischemic stroke. We showed that IL-4-polarized BV2 cells may promote the tube formation in vitro and angiogenesis in vivo through the secretion of exosomes containing miRNA-26a. Moreover, exosomes released by IL-4-polarized microglia may have a potential therapeutic value in the treatment of stroke.

Current stroke therapies include regulatory $\mathrm{T}$ cells, ${ }^{18}$ BMDM, ${ }^{19}$ human mesenchymal stem cells, ${ }^{20}$ and neural stem cells, ${ }^{21}$ all of which might have the potential to shift the inflammatory environment and restore the neurological function after a stroke. However, as a kind of resident macrophages, microglia could be polarized or stimulated directly by the changes in the microenvironment in the brain during all stages of a stroke. Angiogenesis is essential in physiological processes, such as embryonic development and wound-healing tissue repair. ${ }^{22}$ New capillaries are formed from pre-existing blood vessels, allowing the recovery of the supply of anti-inflammatory factors and neuron growth factors. Microglia/macrophages are not only key players in inflammatory diseases, but also in promoting angiogenesis. ${ }^{23}$ In this work, our results showed that microglia could promote angiogenesis not by conventional pathways (i.e., direct cell-cell contact or VEGF signaling), but by secreting exosomes, which concurs with previous reports. ${ }^{24-26}$

The proteomic analysis of exosomes has revealed the presence of specific proteins involved in cell motility, angiogenesis, inflammatory regulation, or neuromodulation, suggesting that glial cells use unconventional pathways for protein secretion. ${ }^{27}$ Some reports have shown that the overexpression of CXCR4 in exosomes secreted from mesenchymal stem cells (ExoCR4) also promotes the recovery of cardiac functions after myocardial infarction (MI). ${ }^{25,28}$ Other reports have also shown that the exosome miRNAs from breast cancer (let-7a, miR-23b, miR-27a/b, miR-21, let-7, and miR-320b) are known to present anti-angiogenic activity. ${ }^{29}$ In our study, the expression of miRNA-26a in M0, M1 and M2 microglia was quite different, and there have been numerous reports showing that miRNA-26a is closely related to angiogenesis. Qian et al. and other authors found that miRNA-26a promoted tumor growth and angiogenesis in glioma by directly targeting prohibitin. ${ }^{17}$ On the other hand, miRNA-26a suppresses the epithelial mesenchymal transition in human hepatocellular carcinoma; miRNA-26a is also a key factor in angiogenesis in diabetic wound healing. ${ }^{30,31}$ These results indicate that different exosomes from of various cell types have different effects on angiogenesis under special circumstances. ${ }^{32}$

The hypoxic state is a situation in which exosome-mediated signaling promotes angiogenesis in some solid tumors and ischemic infarction. ${ }^{32-34}$ In the neural system, the hypoxia-inducible factor (HIF) pathway is involved in angiogenesis in an exosome-dependent manner. ${ }^{35}$ The study of exosomes provided a platform for the diagnosis and monitoring of neurodegenerative progression. ${ }^{36}$ In our experiment, the M2-like microglia could secrete specific exosomes to promote neovascularization, and then carry more Th2/M2-type cells into the ischemic region, which would be beneficial in the recovery from ischemic stroke. 
We injected the M1-type microglia into mice along with the M2-type microglia, but the mice died rapidly, perhaps due to strong inflammatory responses. The HIF pathway may be involved in the pro-angiogenic activity of the exosomes secreted from the M2 microglia.

Taken together, our data demonstrates that IL-4-polarized microglia could ameliorate the damage caused by ischemic stroke by promoting angiogenesis through the secretion of exosomes. Still, the exact mechanisms of how IL-4-polarized microglia secrete these exosomes or how these exosomes help in recovery still need further investigation. ${ }^{37}$ The exact contents of these exosomes and the potential effects of this substance need to be elucidated as well. Nevertheless, our current data suggests that using exosomes derived from IL-4-polarized microglia may be considered a novel therapeutic method for the treatment of ischemic stroke.

\section{References}

1. Fan Y, Xie L, Chung CY. Signaling pathways controlling microglia chemotaxis. Mol Cells. 2017;40(3):163-168.

2. Kanazawa M, Miura M, Toriyabe $M$, et al. Microglia preconditioned by oxygen-glucose deprivation promote functional recovery in ischemic rats. Sci Rep. 2017;7:42582.

3. Narantuya D, Nagai A, Sheikh AM, et al. Human microglia transplanted in rat focal ischemia brain induce neuroprotection and behavioral improvement. PLOS ONE. 2010;5(7):e11746.

4. Hu X, Li P, Guo Y, et al. Microglia/macrophage polarization dynamics reveal novel mechanism of injury expansion after focal cerebral ischemia. Stroke. 2012;43(11):3063-3070.

5. Lee JA, Song HY, Ju SM, et al. Suppression of inducible nitric oxide synthase and cyclooxygenase-2 by cell-permeable superoxide dismutase in lipopolysaccharide-stimulated BV-2 microglial cells. Mol Cells. 2010;29(3):245-250.

6. Korhonen P, Kanninen KM, Lehtonen S, et al. Immunomodulation by interleukin-33 is protective in stroke through modulation of inflammation. Brain Behav Immun. 2015;49:322-336.

7. Liu X, Liu J, Zhao S, et al. Interleukin- 4 is essential for microglia/macrophage M2 polarization and long-term recovery after cerebral ischemia. Stroke. 2016;47(2):498-504.

8. Amantea D, Certo M, Petrelli F, et al. Azithromycin protects mice against ischemic stroke injury by promoting macrophage transition towards M2 phenotype. Exp Neurol. 2016;275(Pt 1):116-125.

9. Chernykh ER, Shevela EY, Starostina NM, et al. Safety and therapeutic potential of M2-macrophages in stroke treatment. Cell Transplant. 2015;25(8):1461-1471.

10. Desestret V, Riou A, Chauveau F, et al. In vitro and in vivo models of cerebral ischemia show discrepancy in therapeutic effects of $M 2$ macrophages. PLOS ONE. 2013;8(6):e67063.

11. Fumagalli S, Perego C, Pischiutta F, Zanier ER, De Simoni MG. The ischemic environment drives microglia and macrophage function. Front Neurol. 2015;6:81.

12. Xin H, Li Y, Cui Y, Yang JJ, Zhang ZG, Chopp M. Systemic administration of exosomes released from mesenchymal stromal cells promote functional recovery and neurovascular plasticity after stroke in rats. J Cereb Blood Flow Metab. 2013;33(11):1711-1715.

13. Jin $Q$, Cheng J, Liu Y, et al. Improvement of functional recovery by chronic metformin treatment is associated with enhanced alternative activation of microglia/macrophages and increased angiogenesis and neurogenesis following experimental stroke. Brain Behav Immun. 2014;40:131-142.

14. Fan $Y$, Xiong $X$, Zhang $Y$, et al. MKEY, a peptide inhibitor of $C X C L 4$ CCL5 heterodimer formation, protects against stroke in mice. $J \mathrm{Am}$ Heart Assoc. 2016;5(9):e003615.

15. Nie L, Wang S, Wang X, et al. In vivo volumetric photoacoustic molecular angiography and therapeutic monitoring with targeted plasmonic nanostars. Small. 2014;10(8):1585-1593.
16. Nie L, Huang P, Li W, et al. Early-stage imaging of nanocarrierenhanced chemotherapy response in living subjects by scalable photoacoustic microscopy. ACS Nano. 2014;8(12):12141-12150.

17. Qian X, Zhao P, Li W, et al. MicroRNA-26a promotes tumor growth and angiogenesis in glioma by directly targeting prohibitin. CNS Neurosci Ther. 2013;19(10):804-812.

18. Li P, Mao L, Zhou G, et al. Adoptive regulatory T-cell therapy preserves systemic immune homeostasis after cerebral ischemia. Stroke. 2013; 44(12):3509-3515.

19. Jiang C, Wang J, Yu L, et al. Comparison of the therapeutic effects of bone marrow mononuclear cells and microglia for permanent cerebral ischemia. Behav Brain Res. 2013;250:222-229.

20. Wang $H$, Nagai A, Sheikh AM, et al. Human mesenchymal stem cell transplantation changes proinflammatory gene expression through a nuclear factor-kappaB-dependent pathway in a rat focal cerebral ischemic model. J Neurosci Res. 2013;91(11):1440-1449.

21. Patkar S, Tate R, Modo M, Plevin R, Carswell HV. Conditionally immortalized neural stem cells promote functional recovery and brain plasticity after transient focal cerebral ischemia in mice. Stem Cell Res. 2012;8(1):14-25.

22. Arai $\mathrm{K}$, Jin G, Navaratna D, Lo EH. Brain angiogenesis in developmental and pathological processes: Neurovascular injury and angiogenic recovery after stroke. FEBS J. 2009;276(17):4644-4652.

23. Jetten N,Verbruggen S, Gijbels MJ,PostMJ,DeWintherMP,Donners MM. Anti-inflammatory M2, but not pro-inflammatory M1 macrophages promote angiogenesis in vivo. Angiogenesis. 2014;17(1):109-118.

24. Chen J, Ning R, Zacharek A, et al. MiR-126 contributes to human umbilical cord blood cell-induced neurorestorative effects after stroke in type-2 diabetic mice. Stem Cells. 2016;34(1):102-113.

25. Kang K, Ma R, Cai W, et al. Exosomes secreted from CXCR4 overexpressing mesenchymal stem cells promote cardioprotection via Akt signaling pathway following myocardial infarction. Stem Cells Int. 2015;2015:659890.

26. Mineo M, Garfield SH, Taverna S, et al. Exosomes released by K562 chronic myeloid leukemia cells promote angiogenesis in a Src-dependent fashion. Angiogenesis. 2012;15(1):33-45.

27. Lazar I, Clement E, Ducoux-Petit M, et al. Proteome characterization of melanoma exosomes reveals a specific signature for metastatic cell lines. Pigment Cell Melanoma Res. 2015;28(4):464-475.

28. Gleissner CA, Shaked I, Little KM, Ley K. CXC chemokine ligand 4 induces a unique transcriptome in monocyte-derived macrophages. J Immunol. 2010;184(9):4810-4818.

29. Hannafon BN, Carpenter KJ, Berry WL, Janknecht R, Dooley WC, Ding WQ. Exosome-mediated microRNA signaling from breast cancer cells is altered by the anti-angiogenesis agent docosahexaenoic acid (DHA). Mol Cancer. 2015;14:133.

30. Ma DN, Chai ZT, Zhu XD, et al. MicroRNA-26a suppresses epithelial-mesenchymal transition in human hepatocellular carcinoma by repressing enhancer of zeste homolog 2. J Hematol Oncol. 2016;9:1.

31. Zgheib C, Liechty KW. Shedding light on miR-26a: Another key regulator of angiogenesis in diabetic wound healing. J Mol Cell Cardiol. 2016;92:203-205.

32. Garcia NA, Ontoria-Oviedo I, Gonzalez-King H, Diez-Juan A, Sepulveda P. Glucose starvation in cardiomyocytes enhances exosome secretion and promotes angiogenesis in endothelial cells. PLOSONE. 2015;10(9):e0138849.

33. King HW, Michael, MZ, Gleadle JM. Hypoxic enhancement of exosome release by breast cancer cells. BMC Cancer. 2012;12:421.

34. Kucharzewska P, Christianson HC, Welch JE, et al. Exosomes reflect the hypoxic status of glioma cells and mediate hypoxia-dependent activation of vascular cells during tumor development. Proc Natl Acad SciU S A. 2013;110(18):7312-7317.

35. Mayo JN, Bearden SE. Driving the hypoxia inducible pathway in human pericytes promotes vascular density in an exosome dependent manner. Microcirculation. 2015;22(8):711-723.

36. Taylor DD, Gercel-Taylor C. Exosome platform for diagnosis and monitoring of traumatic brain injury. Philos Trans R Soc Lond B Biol Sci. 2014; 369(1652). doi:10.1098/rstb.2013.0503

37. Xia CY, Zhang S, Gao Y, Wang ZZ, Chen NH. Selective modulation of microglia polarization to M2 phenotype for stroke treatment. Int Immunopharmacol. 2015;25(2):377-382. 